\title{
Conocimientos sobre salud bucodental y evaluación de higiene oral antes y después de una intervención educativa en niños de 9-10 años
}

\section{Oral health knowledge and tooth brushing quality before and after an educational intervention in children aged 9 and 10}

\author{
Bosch Robaina R*, Rubio Alonso M**, García Hoyos F***
}

\section{RESUMEN}

Introducción: La salud bucodental infantil es un objetivo de primer orden en el contexto de las políticas de salud pública. El objetivo de este estudio es analizar los conocimientos sobre salud bucodental y nivel de higiene oral antes y después de una intervención educativa.

Material y métodos: Se incluyeron en el estudio 50 niños de 9-10 años de edad. Los escolares realizaron una encuesta sobre conocimientos acerca de la salud bucodental antes y después de la intervención educativa que consistió en tres charlas participativas en grupos de 25 niños. Se evaluó el índice de placa de O'Leary antes y después de la intervención educativa.

Resultados: En la encuesta realizada antes de la intervención el $78 \%$ contestaron que sabían en qué consiste la caries y cómo prevenirla. En cambio el $60 \%$ contestó que sólo había que acudir al dentista cuando hubiera algún problema bucal. Antes de la intervención educativa, el $80 \%$ de los niños tenían un índice de O’Leary superior al $20 \%$. Se obtuvieron cambios significativos en cuanto a conocimientos de salud bucal así como en la calidad del cepillado después de la intervención educativa.

Conclusiones: Los conocimientos de los niños sobre salud bucodental así como la calidad del cepillado dental mejoraron significativamente después de la intervención educativa.

Palabras clave: Salud bucodental, niños, intervención educativa.

\section{SUMMARY}

Introduction: Children's oral health is an essential target for public health policies. The aim of this study is to analyze the oral health knowledge and the quality of oral hygiene before and after an educational intervention. Material and Methods: Primary school fifty children aged 9 and 10 were included in the study. They conducted a survey on oral health before and after an intervention including three educational workshops. Oral hygiene O'Leary index was evaluated before and after the intervention.

Results: In the survey conducted before the intervention, $78 \%$ said they knew what tooth decay was and how they could prevent it. In contrast, $60 \%$ answered that they only had to go to the dentist just when an oral problem appeared. Before the educational intervention, $80 \%$ of children had an O'Leary index above $20 \%$.

* $\quad$ Estudiante de Licenciatura de Odontología. Facultad de Ciencias Biomédicas. Universidad Europea de Madrid.

** Departamento de Especialidades Médicas. Facultad de Ciencias Biomédicas. Universidad Europea de Madrid.

*** Departamento de Odontología. Facultad de Ciencias Biomédicas. Universidad Europea de Madrid 
After the educational intervention, significant changes were obtained for knowledge about oral health and the quality of brushing.

Conclusions: The children knowledge about oral health and the quality of tooth brushing improved significantly after the educational intervention.

Key words: Oral health, children, educational intervention..

Fecha de recepción: 28 de junio de 2010.

Aceptado para publicación: 26 de julio de 2010.

Bosch Robaina R, Rubio Alonso M, García Hoyos F. Conocimientos sobre salud bucodental y evaluación de higiene oral antes y después de una intervención educativa en niños de 9-10 años. Av. Odontoestomatol 2012; 28 (1): 17-23.

\section{INTRODUCCIÓN}

Las enfermedades bucodentales constituyen un importante problema de salud pública por su alta prevalencia y fuerte impacto sobre las personas y la sociedad en términos de dolor, malestar, limitación y discapacidad social y funcional (1).

Muchos de los factores de riesgo que se asocian a estas enfermedades se relacionan con un inadecuado estilo de vida y unos hábitos insanos adquiridos a lo largo del tiempo (2). La Educación para la Salud fue definida por la OMS como un campo especial de las Ciencias de la Salud cuyo objetivo es la transmisión de conocimientos y el desarrollo de una conducta encaminada a la conservación de salud del individuo y de la sociedad. La educación es el mecanismo más eficaz del que disponemos para generar un cambio de actitud hacia modos de vida más saludables $(3,4)$.

La salud bucodental infantil es un objetivo de primer orden en el contexto de las políticas de salud modernas en cualquiera de los países de nuestro entorno. El enfoque preventivo y las actividades que se aplican desde los primeros años de vida son claves para el éxito de los programas de salud $(5,6,7)$. En los países desarrollados la caries dental infantil ha disminuido en las últimas décadas. Este descenso se debe a la mejora del nivel de vida, a la implantación de programas preventivos comunitarios y a los avances en la atención odontológica (1). Aún así, en España según los datos de la Encuesta de Salud Oral en España de 2005 a los 12 años sólo el 53 \% de los niños están libres de caries (8).

La promoción de hábitos saludables, la prevención de las principales patologías bucodentales y su detección precoz mediante revisiones periódicas, constituyen un conjunto de acciones de reconocida utilidad en la salud general de los niños ya que estas patologías tienen una alta prevalencia y provocan gran morbilidad (4-8).

Los objetivos del estudio son conocer y comparar la situación sobre conocimientos en salud bucodental y el nivel de higiene oral en niños de 9-10 años, antes y después de realizar una intervención educativa.

\section{MATERIAL Y MÉTODOS}

Se ha realizado un estudio cuasiexperimental de tipo serie temporal usando el mismo grupo de escolares de cuarto de primaria, como control (antes de la intervención) y como experimental (después de la intervención).

Se obtuvo el permiso de la dirección del colegio y el consentimiento informado de la Asociación de Padres de Alumnos (A.P.A.) del colegio para la realización del estudio.

La muestra estuvo integrada por 50 niños del colegio público Los Almendros, del Ayuntamiento de 
Rivas Vaciamadrid (Madrid). Se incluyeron en el estudio todos los niños de 9-10 años que cursaban $4^{\circ}$ de primaria en dicho centro. El único criterio de exclusión fue la presencia de tratamiento con ortodoncia fija.

\section{Primera fase}

Se distribuyó una encuesta sobre conocimientos de salud bucodental. Para la elaboración de la encuesta consultamos distintos modelos de encuestas sobre salud realizadas en España $(6,9,10)$. Se incluyeron las siguientes variables:

- Sexo.

- Frecuencia de visitas al dentista.

- Número de cepillados al día.

- Aprendizaje de cepillado.

- Conocimiento sobre caries dental y prevención.

- Uso de enjuague bucal e importancia.

- Uso de seda dental e importancia.

- Cuidado de las encías.

- Relación entre consumo de dulces y salud bucal.

- Importancia de higiene bucal

- Necesidad de conocimientos sobre higiene bucal.

Posterior a la recogida de datos, se procedió a la evaluación cuantitativa de la higiene bucal tras cepillado dental de los escolares. Esta evaluación se realizó aplicando el índice de O’Leary $(11,12)$. En la determinación de este índice se utilizaron pastillas reveladoras de placa (Plac Dent ${ }^{\circledR}$ ).

Se siguió el siguiente procedimiento:

- Se indicó a los escolares que realizasen el cepillado dental tal y como lo realizaban habitualmente. Para ello se les proporcionó a todos el mismo modelo de cepillo dental infantil y dentífrico con flúor.

- Se les administró una pastilla reveladora y se les indicó que la masticaran y distribuyeran de forma homogénea por todas las superficies dentales.

- Con ayuda del depresor lingual y luz natural se comprobaron las superficies de los dientes que se habían coloreado con la sustancia reveladora y se registró en el diagrama del formulario.

- Se calculó el porcentaje de superficies teñidas respecto al total de superficies examinadas.
Los resultados del índice fueron clasificados de la siguiente forma:

- De 0 a 20\%: bien.

- Del $21 \%$ al $30 \%$ : regular.

— Más del 30 \%: mal.

Se recogieron los datos del total de los niños que participaron en el estudio así como el valor del Índice de O'Leary de cada escolar. Se analizaron los resultados obtenidos.

\section{Segunda fase}

Dos semanas después de la primera recogida de datos se procedió a realizar la intervención educativa sobre salud bucodental. Se realizaron tres charlas educativas de unos 25 minutos de duración cada una, en las que se utilizó material audiovisual adaptado a la edad de los niños.

Las charlas educativas impartidas a los escolares se realizaron en dos grupos de 25 niños cada uno. En las mismas se tocaron temas generales sobre la boca, sus estructuras y funciones. Posteriormente se mostraron algunas de las enfermedades que pueden afectar la boca (caries y gingivitis) y se explicaron conceptos básicos sobre su patogenia y evolución. Asimismo se explicó a los escolares como prevenir esas enfermedades y los medios para hacerlo (cepillado dental, seda dental y colutorios). Se realizaron actividades prácticas (demostración de técnica de cepillado con tipodonto y juegos de preguntas y respuestas) en cada actividad.

\section{Tercera fase}

Una semana después de la intervención educativa se procedió a la aplicación de una segunda encuesta. Se recogieron las mismas variables que en la encuesta de la primera fase.

Posteriormente se volvió a medir el índice de $\mathrm{O}^{\prime}$ Leary tras el cepillado dental. Se les indicó a los escolares que tuvieran en cuenta lo aprendido durante la intervención.

Para el análisis de los datos se utilizó el programa SPSS 17. Para analizar diferencias entre niños y niñas 
en las respuestas a la primera encuesta se utilizó la prueba de chi cuadrado de Pearson. Para comparar los resultados obtenidos en la $1^{\mathrm{a}}$ y $2^{\mathrm{a}}$ encuesta se utilizó la prueba de McNemar y la prueba de los rangos con signo de Wilcoxon. Se consideraron estadísticamente significativos valores de $p$ menores de 0,05 .

\section{RESULTADOS}

Se incluyeron 50 escolares de los cuales 31 (62\%) eran varones. En la encuesta realizada en la primera fase encontramos los siguientes resultados: 39 escolares (78\%) contestaron que conocían en qué consiste la caries y también 39 (78\%) pensaban que se podía prevenir. El $76 \%$ de los niños contestaron que habían sido sus padres los que les habían enseñado a cepillarse los dientes, mientras que el $24 \%$ restante contestaron que les había enseñado el odontólogo. En cuanto a la frecuencia de visitas al odontólogo el $60 \%$ (30 escolares) contestaron que había que acudir cuando se presentara algún problema en la boca y el resto (20 escolares) que había que acudir al menos una vez al año.

El 82\% (41 niños) contestó que comer dulces se relacionaba con problemas en los dientes, 7 (14\%) contestaron que no había ninguna relación, uno contestó que eran buenos para los dientes y uno de los encuestados no contestó a la pregunta. En cuanto al sangrado de encías el 64\% (32 niños) contestaron que indicaba que las encías estaban enfermas mientras que el $36 \%$ restante contestó que era algo normal. El 50\% de los niños contestaron que convenía cepillarse los dientes 2 veces al día, el 28\% (14 niños) contestaron 3 o más veces y el 22\% (11 niños) una sola vez al día. En cuanto al uso de seda dental y colutorio contestaron que sí convenía usarlos el 30\% (15 niños) y el $76 \%$ de los niños respectivamente.

El 84\% de los encuestados contestaron que consideraban la higiene oral algo muy importante y el $16 \%$, importante. A la pregunta ¿Te gustaría aprender a cuidar mejor tus dientes?, el 94\% (47 niños) contestaron que sí.

Los resultados de la evaluación de la higiene oral mediante pastillas reveladoras fueron los siguientes: la mediana de puntuación (índice de $\mathrm{O}$ 'Leary) fue $32 \%$ con un rango entre $6 \%$ y $48 \%$. Diez niños obtuvieron una puntuación entre 0 y $20 \%$, (bien) 10 niños una puntuación entre 21 y 30\% (regular) y 30 niños una puntuación mayor de 30\% (mal).

Realizamos una comparación de los resultados obtenidos en niños y niñas y encontramos diferencias estadísticamente significativas en dos de las variables recogidas en la encuesta: el 63,2\% de las niñas contestaron que convenía ir al dentista al menos una vez al año, frente a un $25,8 \%$ de niños $(p=0,009)$. De las niñas el $52,6 \%$ contestaron que debían cepillarse los dientes 3 veces al día y el 47,4\% dos veces. De los niños el 12,9\% contestó tres veces al día, el 51,6\% dos veces y el $22 \%$ contestó una sola vez al día $(p=0,001)$.

El análisis comparativo de los datos obtenidos en las encuestas antes y después de la intervención educativa se muestra en las tablas 1 y 2 .

Las diferencias en los resultados del índice de O Leary clasificados en "bien" (puntuación entre 0 y 20\%), "regular" (entre 21 y 30\%) y "mal" (más de $30 \%$ ) se muestran en la tabla 3 . Observamos una diferencia estadísticamente significativa en los resultados obtenidos antes y después de la intervención educativa.

\section{DISCUSIÓN}

Existen varios estudios publicados con objetivos similares al realizado por nosotros (13-17). En 2005 se publicó un estudio muy similar realizado en $\mathrm{Ma}$ drid (14). En este estudio, al igual que en los antes mencionados, obtuvieron resultados que confirman la utilidad de una intervención educativa en escolares.

En el presente estudio se observó que la mayoría de los escolares tenían conocimientos básicos sobre educación bucodental. Antes de la intervención educativa, el $78 \%$ afirmaban saber en qué consiste la caries dental y también el $78 \%$ pensaba que se podía prevenir. Asimismo el $82 \%$ asociaba la ingesta de dulces con caries. Estos resultados difieren de otros estudios en los que se observa un nivel de conoci- 


\begin{tabular}{|c|c|c|c|}
\hline $\begin{array}{r}\text { TABLA } 1 . \\
\text { GENERALES SOBR } \\
\text { RESPUESTAS DE L } \\
\text { (ENCUESTA 1) Y D } \\
\text { DE LA INTERV }\end{array}$ & $\begin{array}{l}\text { CONOC } \\
\text { E SALUD } \\
\text { OS ESCC } \\
\text { ESPUÉS } \\
\text { NCIÓN E }\end{array}$ & $\begin{array}{l}\text { MIENTOS } \\
\text { BUCODE } \\
\text { LARES A } \\
\text { (ENCUES } \\
\text { DUCATIV }\end{array}$ & $\begin{array}{l}\text { NTAL. } \\
\text { NTES } \\
\text { TA 2) } \\
\text { A }\end{array}$ \\
\hline & Encl & lesta & \\
\hline & $\begin{array}{c}1 \\
N(\%)\end{array}$ & $\begin{array}{c}2 \\
N(\%)\end{array}$ & $p$ \\
\hline $\begin{array}{l}\text { Conocimiento caries }{ }^{1} \\
\text { - Sí }\end{array}$ & 39 (78\%) & $48(96 \%)$ & 0,02 \\
\hline - No & $11(22 \%)$ & $2(4 \%)$ & \\
\hline Prevención caries ${ }^{2}$ & & & 0,02 \\
\hline • Sí & $39(78 \%)$ & $48(96 \%)$ & \\
\hline - No & $11(22 \%)$ & $2(4 \%)$ & \\
\hline Frecuencia asistencia & & & - \\
\hline $\begin{array}{l}\text { - Cuando haya algún } \\
\text { problema }\end{array}$ & $\begin{array}{l}30(60 \%) \\
20(40 \%)\end{array}$ & $\begin{array}{r}6(12 \%) \\
44(88 \%)\end{array}$ & $<0,001$ \\
\hline Mi menos la vez/anio & $20(40 \%)$ & $44(00 \%)$ & \\
\hline $\begin{array}{l}\text { Relación dulces-salud } \\
\text { oral }^{4}\end{array}$ & & & $<0,001$ \\
\hline - Producen problemas & $41(82 \%)$ & $49(98 \%)$ & \\
\hline • No hay relación & $7(14 \%)$ & $1(2 \%)$ & \\
\hline - Son buenos & $1(2 \%)$ & 0 & \\
\hline $\begin{array}{l}\text { Sangrado de encías } \\
\text { - Indica que están } \\
\text { enfermas } \\
\text { - Es normal }\end{array}$ & $\begin{array}{l}32(64 \%) \\
18(36 \%)\end{array}$ & $\begin{array}{c}48(96 \%) \\
2(4 \%)\end{array}$ & $<0,001$ \\
\hline $\begin{array}{l}\text { ¿Sabes qué es la caries dental? } \\
\text { ¿Crees que la caries se puede } \\
\text { ¿Cuándo crees que debes ir al } \\
\text { ¿Qué tiene que ver comer dulc } \\
\text { Si te sangran las encías al cepi }\end{array}$ & $\begin{array}{l}r ? \\
\text { ista? }\end{array}$ & la boca? & \\
\hline
\end{tabular}

mientos inferior antes de las intervenciones educativas $(14,15,17)$.

En cuanto a la relación entre género de los escolares y algunas de las variables estudiadas nuestros resultados reflejan que hay un porcentaje mayor de niñas

\begin{tabular}{|c|c|c|c|}
\hline \multicolumn{4}{|c|}{$\begin{array}{l}\text { TABLA 2.- CONOCIMIENTOS SOBRE } \\
\text { HÁBITOS HIGIENICOS E IMPORTANCIA DE } \\
\text { LA SALUD BUICODENTAL. RESPUIESTAS DE } \\
\text { LOS ESCOLARES ANTES (ENCUESTA 1) Y } \\
\text { DESPUÉS (ENCUESTA 2) DE LA } \\
\text { INTERVENCIÓN EDUCATIVA }\end{array}$} \\
\hline & \multicolumn{2}{|c|}{ Encuesta } & \multirow[b]{2}{*}{$p$} \\
\hline & $\begin{array}{c}1 \\
N(\%)\end{array}$ & $\begin{array}{c}2 \\
N(\%)\end{array}$ & \\
\hline $\begin{array}{l}N^{0} \text { cepillados/día }{ }^{1} \\
\text { - } 1 \text { vez } \\
\text { - } 2 \text { veces } \\
\text { - } 3 \text { o más } \\
\text { Seda dental } \\
\text { - Sí } \\
\text { - No } \\
\text { Colutorio } \\
\text { - Sí } \\
\text { - No } \\
\text { Importancia higiene } \\
\text { - Muy importante } \\
\text { - Importante } \\
\text { - Poco importante } \\
\text { Aprender más } \\
\text { - Sí } \\
\text { - No }\end{array}$ & $\begin{array}{c}11(22 \%) \\
25(50 \%) \\
14(28 \%) \\
15(30 \%) \\
35(70 \%) \\
38(76 \%) \\
12(24 \%) \\
42(84 \%) \\
8(16 \%) \\
00 \\
47(94 \%) \\
3(6 \%)\end{array}$ & $\begin{array}{c}4(8 \%) \\
6(12 \%) \\
40(80 \%) \\
50(100 \%) \\
0 \\
50(100 \%) \\
0 \\
46(92 \%) \\
4(8 \%)\end{array}$ & $<0,001$ \\
\hline
\end{tabular}

${ }^{1}$ ¿Cuántas veces hay que cepillarse los dientes al día?

2 ¿Hay que utilizar la seda dental para la limpieza de los dientes?

¿Hay que utilizar algún Colutorio?

${ }^{4}$ ¿Qué importancia le darías a mantener una buena higiene de la boca y mantener tus dientes sanos?

¿Te gustaría aprender más sobre cómo cuidar tus dientes?

TABLA 3.-RESULTADOS DEL ÍNDICE DE O'LEARY ANTES Y DESPUÉS DE LA INTERVENCIÓN EDUCATIVA

\begin{tabular}{|l|c|c|c|}
\hline & $\begin{array}{c}\text { Antes } \\
\text { N (\%) }\end{array}$ & $\begin{array}{c}\text { Después } \\
\text { N (\%) }\end{array}$ & $p$ \\
\hline Bien & $10(20 \%)$ & $24(48 \%)$ & \\
Regular & $10(20 \%)$ & $16(32 \%)$ & 0,001 \\
Mal & $30(60 \%)$ & $10(20 \%)$ & \\
\hline
\end{tabular}

Bien: indice de O'Leary hasta $20 \%$

Regular: de $21 \%$ a $30 \%$

Mal: superior a $30 \%$ 
que de niños que contestan que hay que acudir al menos una vez al año al odontólogo y que hay un número significativamente mayor de niñas que contestan que hay que cepillarse los dientes 2 ó 3 veces al día. Estos resultados coinciden con los de otros estudios publicados $(14,16,17)$.

La mayoría de los niños (76\%) contestaron que habían sido sus padres lo que les habían enseñado a cepillarse los dientes. Puesto que los resultados en la evaluación de la higiene oral mediante pastillas reveladoras de placa, no fueron muy buenos (40 niños de los 50 estudiados obtuvieron un índice mayor del 20\%), esto nos hace pensar que probablemente los padres no dispongan de la información ni los medios adecuados para enseñar una técnica correcta y eficiente de cepillado a los niños. Esto invitaría a analizar en próximos estudios el conocimiento de los padres sobre salud bucal y cómo transmiten estos conocimientos a sus hijos. En nuestro caso se ofreció la posibilidad de brindar instrucción a los padres o tutores pero no fue aceptada por su parte.

Un aspecto importante, y que ninguno de los estudios revisados incluye dentro de su cuestionario, es la motivación de los niños por los conocimientos de salud bucodental. En nuestro estudio incluimos una pregunta sobre la importancia que le otorgaban a la salud bucodental (el $84 \%$ en la $1^{\text {a }}$ encuesta y el $92 \%$ en la $2^{a}$, contestó que era muy importante) y una pregunta sobre su motivación para aprender sobre este tema (el $94 \%$ en la $1^{a}$ encuesta y el $98 \%$ en la $2^{a}$ contestaron que querían aprender más). Este resultado muestra una preocupación de los escolares por su salud bucal y una gran motivación para aprender, antes y después de la intervención educativa, por lo que sería conveniente recomendar un fomento de actividades educativas como forma de inculcar conocimientos básicos que unos niños de esta edad deberían tener adquiridos.

En cuanto a la evaluación cuantitativa de la calidad del cepillado nuestro estudio coincide con los resultados obtenidos por otros investigadores $(13,15-20)$ que confirman la utilidad de una intervención educativa al mostrar una evolución estadísticamente significativa en cuanto a la mejoría de la calidad del cepillado en los escolares.
Es importante reseñar que la actitud receptiva y la atención mostrada por los escolares fueron muy satisfactorias lo cual constituyó un aspecto determinante en los resultados obtenidos. Además, la respuesta de los profesores en todo momento sobrepasó nuestras expectativas iniciales, lo cual contribuyó sobremanera a la calidad de la intervención.

Finalmente, observando los resultados obtenidos, resaltamos la importancia de este tipo de intervenciones educativas en estas edades y proponemos que se incluyan dentro de la programación escolar.

\section{BIBLIOGRAFÍA}

1. Gestal JJ, Smyth E, Cuenca E, Montes A. Epidemiología y prevención de las enfermedades de la cavidad oral: caries dental, periodontopatías y cáncer. En: Piédrola Gil. Medicina Preventiva y Salud Pública. Elsevier Masson: Barcelona; 2008.

2. Domínguez Rojas V, Astasio Arbiza P, Ortega Molina P, Gordillo Florencio E, García Núñez JA, Bascones Martínez A. Analysis of several risks factors envolved in dental caries trough multiple logistic regresión. International Dental Journal, 1993;43:149-56.

3. Salleras L, Fuentes M, Prat Marín A, Garrido P. Educación sanitaria . Conceptos y métodos. En: Piédrola Gil. Medicina Preventiva y Salud Pública. Elsevier Masson: Barcelona; 2008.

4. Rioboo García R. Educación para la Salud Bucodental. Avances. Odontología Preventiva y Odontología Comunitaria. Madrid, 2002: 1321-41.

5. Junta de Extremadura. Consejería de Bienestar Social. Dirección General de Salud Pública. Programa de Salud Buco-dental. Badajoz 1998.

6. Administración de la Comunidad Autónoma del País Vasco. Departamento de Sanidad y Consumo. Estudio Epidemiológico de la Salud Bucodental infantil en la Comunidad Autónoma Vasca, 1990. 
7. Boletín Oficial del Estado. Disposiciones Generales. Ministerio de Sanidad y Consumo. Num. 243. Secc. 1. Pág. 84823. 2009.

8. Bravo-Pérez M, Casals-Peidró E, CortésMartinicorena FJ, Llodra-Calvo JC, Álvarez-Arenas Pardina I, Hermo-Señariz P et al. Encuesta de Salud Oral en España 2005. RCOE [revista en la Internet]. 2006 [citado 2010 Jun 17];11(4):409-56. Disponible en: http://scielo.isciii.es/scielo.php?script =sci_arttextEpid=S1138-123X2006000400002E $\operatorname{lng}=\mathrm{es}$

9. Biglino I, Coronado A, Gil E, Gil J, Prieto A, Regidor E (1994). Encuesta Nacional de Salud 1993. Rev San Hig Pública. 1994;68:121-78.

10. López Bermejo MA, Samara Shukeir G, Reyes Serrano, Oteo Muñoz C, Cerón Vivancos J. Encuesta sobre Hábitos, Actitudes y Conocimientos sobre Higiene Oral en Niños de 6-7 Años de Edad de la CAM. Odontol Pediatr. 2002;10:920.

11. O' Leary T, Drake R, Naylor. The plaque control record. J Periodontol 1972;43(1):38-9.

12. BL Butler, O Morejón and SB Low. An accurate, time-efficient method to assess plaque accumulation. J Am Dent Assoc 1996;127;1763-6.

13. López Bermejo M.A, González Sanz A, Cerón Vivancos J, Moranz M. Estudio piloto de un programa preventivo bucodental para la población escolar de Madrid. Rev Esp Estomatol 1988;4: 279-90.

14. Caldés Ruisánchez S, Cea Sánchez N, Crespo Aliseda P, Díez Nicolás V, Espino García A, Galán Arévalo S, Albaladejo Vicente R, Domínguez Rojas $\mathrm{V}$. ¿ Una intervención educativa en niños de 12 años de Madrid modifica sus conocimientos y hábitos de higiene buco dental? Av. Odontoestomatol 2005;21(3):149-57.
15. González Ariza S. Promoción y prevención es salud bucal en docentes y escolares de una escuela de Medellín. Revista Colombiana de Investigación en Odontología 2009;1(1):46-57.

16. Agreda et al. Presencia de placa dental en alumnos de quinto grado de la Escuela Básica "Eloy paredes". MedULA 2008;17(2):95-9.

17. Crespo Mafrán, MI, Riesgo Cosme MC, Laffita Lobaina Y, Rodríguez Hung AM, Copello Torres A. Instrucción educativa sobre salud bucal en la Escuela Primaria "Lidia Doce Sánchez". MEDISAN 2010;14(1):232.

18. Livny A, Vered Y, Slouk L, Sgan-Cohen HD. Oral health promotion for schoolchildren - evaluation of a pragmatic approach with emphasis on improving brushing skills. BMC Oral Health 2008, 8:4doi:10.1186/1472-6831-8-4 http://www. biomedcentral.com/1472-6831/8/4.

19. García Álvarez OL, Sexto Delgado NM, Moya Padilla N, López Fernández R. Intervención educativa para el desarrollo de conocimientos sobre salud bucal en la enseñanza primaria. Medisur: Revista electrónica de las Ciencias Médicas en Cienfuegos. 2008;6(2):20-4.

20. Julien MG. The effect of behaviour modification techniques on oral hygiene and gingival health of 10-year-old Canadian Children. Int J Paediatr Dent, 1994;4(1):3-11.

\section{CORRESPONDENCIA}

Margarita Rubio Alonso

Departamento de Especialidades Médicas.

Facultad de Ciencias Biomédicas.

Universidad Europea de Madrid.

Tajo, s/n. Urbanización El Bosque

Villaviciosa de Odón. 28670 Madrid.

e-mail: margarita.rubio@uem.es 